\title{
Tuning Space Mapping Optimization Exploiting Embedded Surrogate Elements
}

\author{
Qingsha S. Cheng ${ }^{\dagger 1}$, John W. Bandler ${ }^{\dagger \diamond 2}$, and Slawomir Koziel ${ }^{\ddagger 3}$ \\ ${ }^{\dagger}$ Department of Electrical \& Computer Engineering, McMaster University, Hamilton, ON, Canada L8S 4K1 \\ ${ }^{\circ}$ Bandler Corporation, Dundas, ON, Canada L9H 5E7 \\ $\ddagger$ School of Science and Engineering, Reykjavik University, Kringlunni 1, IS-103 Reykjavik, Iceland \\ ${ }^{1}$ chengq@mcmaster.ca, ${ }^{2}$ bandler@mcmaster.ca, ${ }^{3}$ koziel@ru.is
}

\begin{abstract}
Inspired by the tuning space mapping concept, the implicit space mapping concept, and surrogate optimization, we propose a simple microwave design optimization/tuning technique. Utilizing co-calibrated ports, our new tuning model is created by substituting sections in the electromagnetic (EM) model with corresponding sections of designable equivalent (surrogate) elements. Several microwave examples demonstrate how the tuning model can be used for design purposes.

Index Terms - Computer-aided design (CAD), engineering optimization, space mapping, surrogate models, design tuning.
\end{abstract}

\section{INTRODUCTION}

Tuning elements $[1,2]$ inserted between "co-calibrated" ports [3,4] have opened doors to new design concepts and technologies in tuning and optimization. Tuning space mapping $[1,2]$ is one of them. However, since these methods currently rely on tuning elements inserted into infinitesimal gaps between the tuning ports, the tuning capacity is limited. At times, negative valued tuning elements may be required. Although not a deterrent to Agilent $\mathrm{ADS}^{1}$, this can pose difficulties to circuit simulators. Often lumped tuning element circuitry has to be inserted in the ports, which requires a translation between the tuning elements and the design parameters. Extra calibration steps are necessary to determine the translation $[1,2]$. Furthermore, the tuning space mapping presented in [1,2] does not easily handle certain cross-sectional parameters such as microstrip line widths and substrate heights.

To address these issues, we propose a surrogate embedding method based on co-calibrated ports. Instead of inserting a tuning element into adjacent ports, we replace an entire section of design interest between the ports with a parameterized surrogate (e.g., empirical microstrip line model with variable length and width, etc.). The resulting structure is designable, fast and accurate. It should have positive design parameters and the ability to model cross-sectional parameters. Replacing large

This work was supported in part by the Natural Sciences and Engineering Research Council of Canada under Grants RGPIN7239-06, STPGP336760-06, and by Bandler Corporation.

${ }^{1}$ Advanced Design System (ADS), Version 2005A, Agilent Technologies, Santa Rosa, CA, 2005. sections of the original structure allows significant tuning capacity. Couplings among and within the remaining structure are maintained. With suitable adjustments of the embedded surrogate elements, the responses of the resulting embedded model (tuning model) can match those of the original structure of interest.

\section{TUNING SPACE MAPPING}

Space mapping $[5,6]$ shifts the CPU burden from an expensive EM simulator (fine model) to a fast equivalent circuit model (coarse model). The concept of simulation-based tuning, also widely used in microwave engineering [3,7], can be considered within the scope of space mapping. In our new tuning space mapping approach, a tuning model of the fine model is constructed by replacing portions of designable interest by suitable surrogate components. The design parameters and preassigned parameters of these surrogate components are chosen to be tunable. In each iteration, to match the fine model, the preassigned parameters of the surrogates are optimized. This process takes little CPU effort as the tuning model is typically implemented within a circuit simulator. With optimal preassigned parameters thus obtained, an updated tuning model is available for design prediction. The prediction is fed back to fine model simulator. This process is repeated until the fine model response is sufficiently close to the design target. The selection of the surrogate elements is as relatively straightforward, or more so, than selecting the tuning elements of $[1,2]$. We simply replace the piece of interest in the fine model with an equivalent distributed circuit model. This way we allow easy tuning of gaps, widths, substrate heights, etc.

\section{THE AlgORITHM}

We are concerned with the following optimization problem

$$
\boldsymbol{x}_{f}^{*}=\arg \min _{\boldsymbol{x}} U\left(\boldsymbol{R}_{f}(\boldsymbol{x})\right)
$$

where $\boldsymbol{R}_{f} \in R^{m}$ denotes the response vector of a fine model of the device of interest, $U$ is a merit function (e.g., a minimax function or a norm), $\boldsymbol{x}$ is a vector of design parameters, and $\boldsymbol{x}_{f}{ }^{*}$ is the optimal solution to be determined. 
In [1,2], the tuning space mapping may involve a tuning model (the fine model with tuning elements inserted into co-calibrated ports [3]), and an additional full-system coarse model or perturbed fine model if a tuning-to-design-parameter conversion is not available. In our proposed method, the tuning model is our initial surrogate. We replace certain designable sub-sections with suitable surrogates. In our examples, they are distributed circuit elements with physical dimensions corresponding to those of the fine model. After a simple parameter extraction procedure, or, rather, an alignment procedure, we match the tuning model with the fine model (the original structure without co-calibrated ports). Some of the fine-model couplings are preserved (or represented through $S$-parameters) in the tuning model. We normally obtain a good surrogate of the fine model. We then tune our tuning model by changing the design parameters of the embedded surrogates to satisfy our goals. The obtained design parameters become our next fine model iterate. A conceptual illustration is shown in Fig. 1.

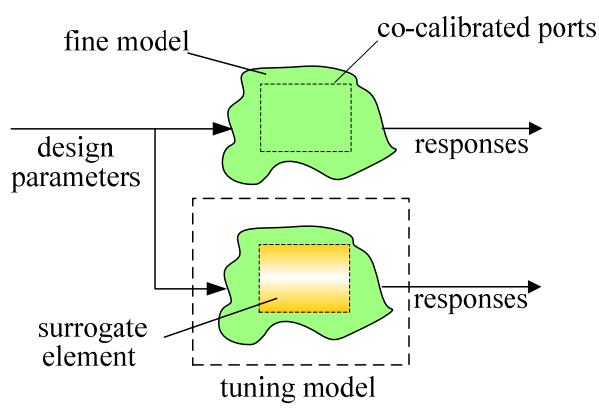

(a)

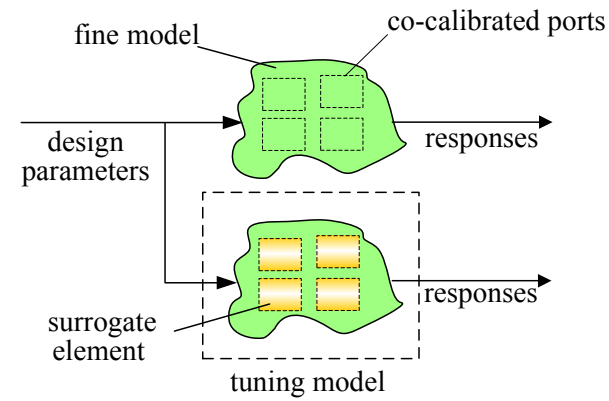

(b)

Fig. 1. Embedded surrogate elements: (a) single element (b) multiple elements.

Our tuning space mapping algorithm produces a sequence of points (design variable vectors) $\boldsymbol{x}^{(i)}, i=0,1, \ldots$. The iteration of the algorithm consists of two steps: alignment of the tuning model with the fine model and the optimization of the tuning model. First, based on fine model (with co-calibrated ports) data at point $\boldsymbol{x}^{(i)}$, the current tuning model $\boldsymbol{R}_{s}^{(i)}$ is built with appropriate surrogate elements replacing certain fine model sections. The tuning model response may not agree with the response of the original fine model at $\boldsymbol{x}^{(i)}$. We align these models by [6]:

$$
\boldsymbol{x}_{p}^{(i)}=\arg \min _{\boldsymbol{x}_{p}}\left\|\boldsymbol{R}_{f}\left(\boldsymbol{x}^{(i)}\right)-\boldsymbol{R}_{s}^{(i)}\left(\boldsymbol{x}^{(i)}, \boldsymbol{x}_{p}\right)\right\|
$$

In the next step, we optimize $\boldsymbol{R}_{s}^{(i)}$ to have it meet the design specifications. We obtain the optimal values of the design parameters $\boldsymbol{x}^{(i+1)}$ as follows [6]:

$$
\boldsymbol{x}^{(i+1)}=\arg \min _{\boldsymbol{x}} U\left(\boldsymbol{R}_{s}^{(i)}\left(\boldsymbol{x}, \boldsymbol{x}_{p}^{(i)}\right)\right)
$$

\section{ILLUSTRATIONS AND EXAMPLES}

\section{A. Coupled Microstrip Line}

We show our tuning method in a coupled microstrip line example with co-calibrated tuning ports simulated in Sonnet $\boldsymbol{e m}^{2}$ (Fig. 2). The length of the coupled lines is $105 \mathrm{mil}$ and the gap between the lines is 5 mil. We obtain a 6-port $S$-parameter file from Sonnet $\boldsymbol{e m}$. To obtain the corresponding 2-port $S$-parameters, we import the $S$-parameter file to Agilent ADS and connect the adjacent port pairs as shown in Fig. 3.

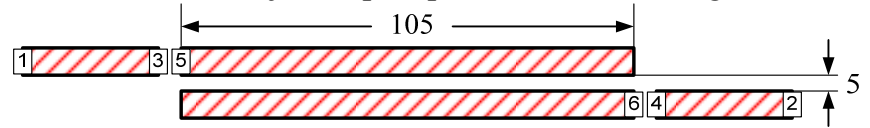

Fig. 2. A coupled microstrip line Sonnet $\boldsymbol{e m}$ model with co-calibrated ports.

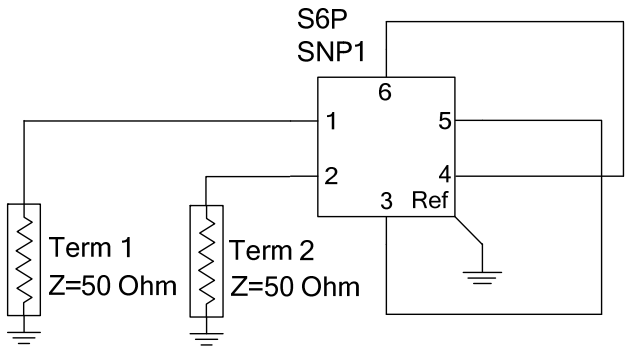

Fig. 3. Importing the 6-port Sonnet $\boldsymbol{e m}$ coupled microstrip line model into Agilent ADS. The adjacent co-calibrated port pairs are connected to obtain its corresponding 2-port $S$-parameters.

We now start to construct our tuning model based on the arrangement of Fig. 3. We short ports 5 and 6 and insert an equivalent circuit microstrip coupled line MCLIN (embedded surrogate) between ports 3 and 4 . We obtain the tuning model shown in Fig. 4.

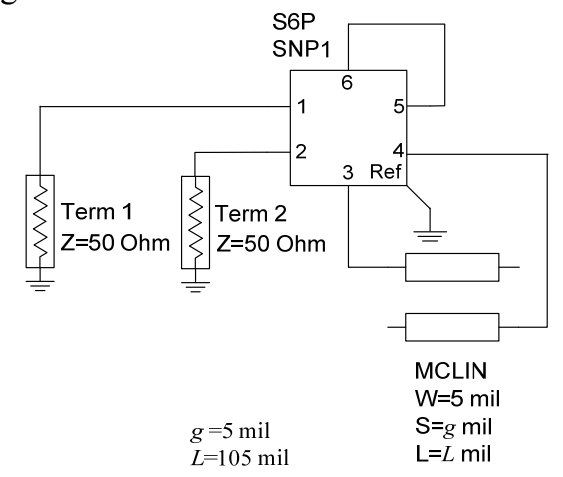

Fig. 4. Tuning model: an equivalent circuit coupled microstrip line (embedded surrogate) replaces the portion of design interest.

\footnotetext{
${ }^{2}$ Sonnet $\boldsymbol{e m}$, Ver. 11.52, Sonnet Software, North Syracuse, NY, 2007.
} 
In the alignment process, we calibrate the dielectric constant and substrate height of the inserted coupled line MCLIN such that the two-port $S$-parameters of the tuning model match that of the fine model. After alignment calibration, we obtained 9.36 for the substrate height and 9.38 for the dielectric constant (originally 10 mil and 9.9, respectively). The match between the fine model and tuning model is very good. For illustration purposes we select our target as a Sonnet em $S$-parameter response for the structure shown in Fig. 5. We optimize the surrogate of Fig. 4 to match this target and obtain a good estimate of the design parameter values as $g=9.78$ and $L=$ 79.58 .

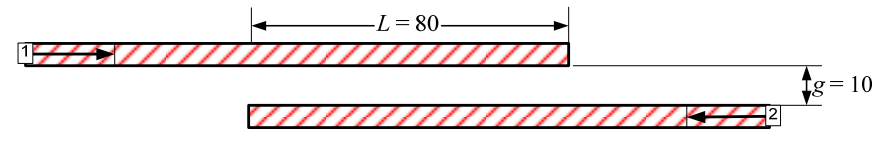

Fig. 5. A coupled microstrip line in Sonnet $\boldsymbol{e m}$ as our target.

\section{B. Three-section Transformer Example}

In this example we demonstrate that our method is capable of designing structures that involve cross-sectional parameters, in this case, widths. This was not easily implemented by the method in $[1,2]$.

Here we demonstrate a 3-section transformer example that involves the design of physical widths and lengths as shown in Fig. 6(a). The design specifications are

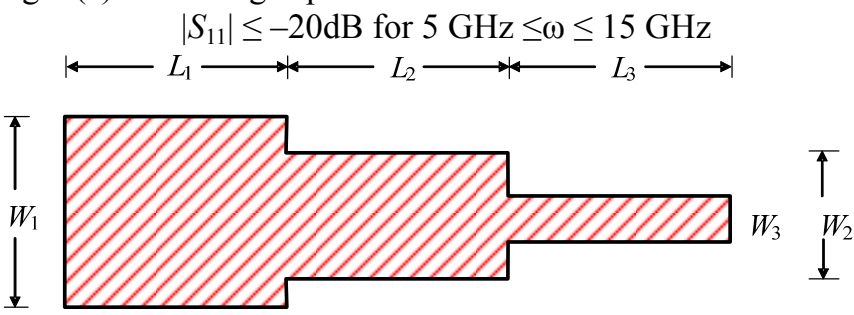

(a)

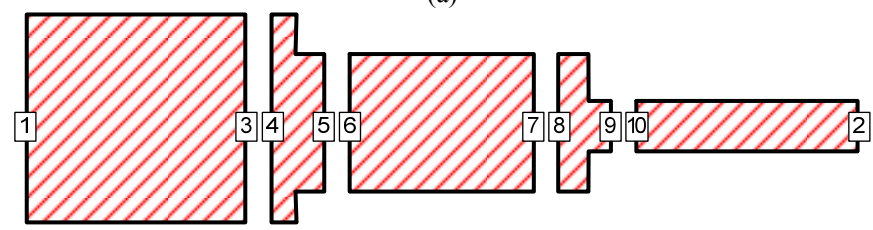

(b)

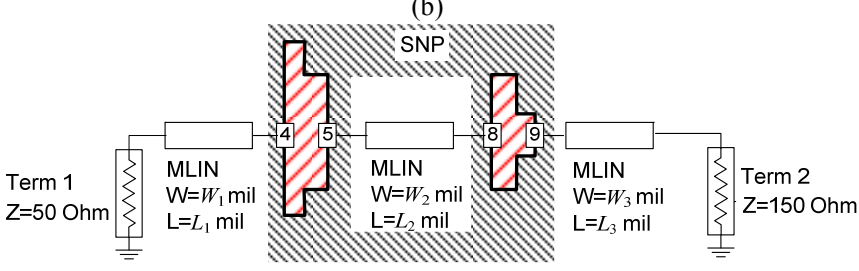

(c)

Fig. 6. The three-section 3:1 microstrip impedance transformer: (a) structure and dimensions; (b) Sonnet $\boldsymbol{e m}$ model with co-calibrated ports; (c) tuning model: replacing the designable components with the ADS model. The hatched area marks what remains in the SNP file.

The model is simulated using Sonnet em. We insert co-calibrated ports inside the structure as demonstrated in Fig. 6(b). The 10-port $S$-parameter S10P file is then imported into ADS. The ADS microstrip transmission line models replace the sections between ports 1 and 4; between 5 and 8; and between 9 and 2 as in Fig 6(c). This should be distinguished from inserting disconnected fine model segments into an equivalent circuit. The tuning model in the ADS schematic is then calibrated against the fine model response by optimizing the preassigned parameters, i.e., the dielectric constants and substrate heights of the microstrip lines. After the model is calibrated, we keep the preassigned parameters as constants and optimize the model in Fig. 6(c) to obtain the next predicted values of the design parameters. In just two iterations, we obtain a good result, as in Fig. 7.

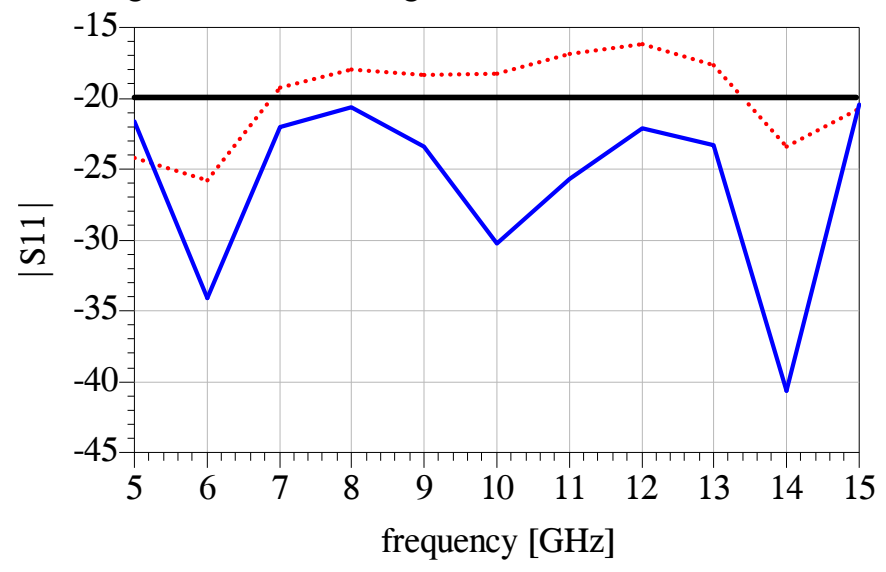

Fig. 7. The three-section 3:1 microstrip impedance transformer Sonnet $\boldsymbol{e m}$ responses: initial (...) and final (-) after two iterations.

\section{Open-loop Ring Resonator Bandpass Filter}

Our third example is the open-loop ring resonator bandpass filter [8] shown in Fig. 8. The design parameters are $\boldsymbol{x}=$ $\left[\begin{array}{lllllll}L_{1} & L_{2} & L_{3} & L_{4} & S_{1} & S_{2} & g\end{array}\right]^{T} \mathrm{~mm}$. Other parameter values are: $W=$ $0.6 \mathrm{~mm}, W_{1}=0.4 \mathrm{~mm}$. The design specifications are

$\left|S_{21}\right| \geq-3 \mathrm{~dB}$ for $2.8 \mathrm{GHz} \leq \omega \leq 3.2 \mathrm{GHz}$
$\left|S_{21}\right| \leq-20 \mathrm{~dB}$ for $1.5 \mathrm{GHz} \leq \omega \leq 2.5 \mathrm{GHz}$
$\left|S_{21}\right| \leq-20 \mathrm{~dB}$ for $3.5 \mathrm{GHz} \leq \omega \leq 4.5 \mathrm{GHz}$

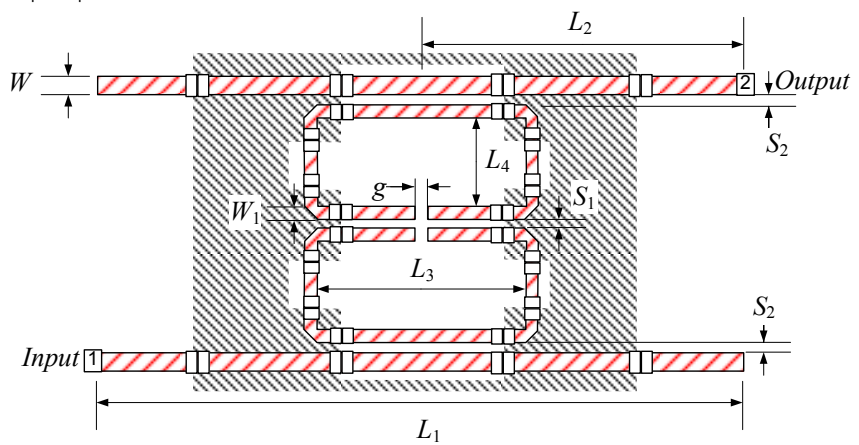

Fig. 8. Open-loop ring resonator bandpass filter: physical structure with co-calibrated ports. Structures outside the hatched area are to be replaced by surrogate elements.

In this example, the fine model is simulated in Sonnet em, the tuning model is constructed and optimized in Agilent ADS. To construct the initial tuning model, in Sonnet $\boldsymbol{e m}$, we first divide the microstrip structure and insert co-calibrated port pairs on the cut edges as shown in Fig. 8. Then, the EM 
structure with the ports is simulated and the resulting SNP data file (50 ports) is imported into the SNP $S$-parameter file component in ADS. Equivalent circuit microstrip lines and coupled line components replace sections of the physical structure in Fig. 9. A new tuning model is now available in the ADS circuit simulator. The design parameters are the same as those of the original design problem $\boldsymbol{x}=\left[\begin{array}{lllllll}L_{1} & L_{2} & L_{3} & L_{4} & S_{1} & S_{2} & g\end{array}\right]^{T}$ $\mathrm{mm}$.

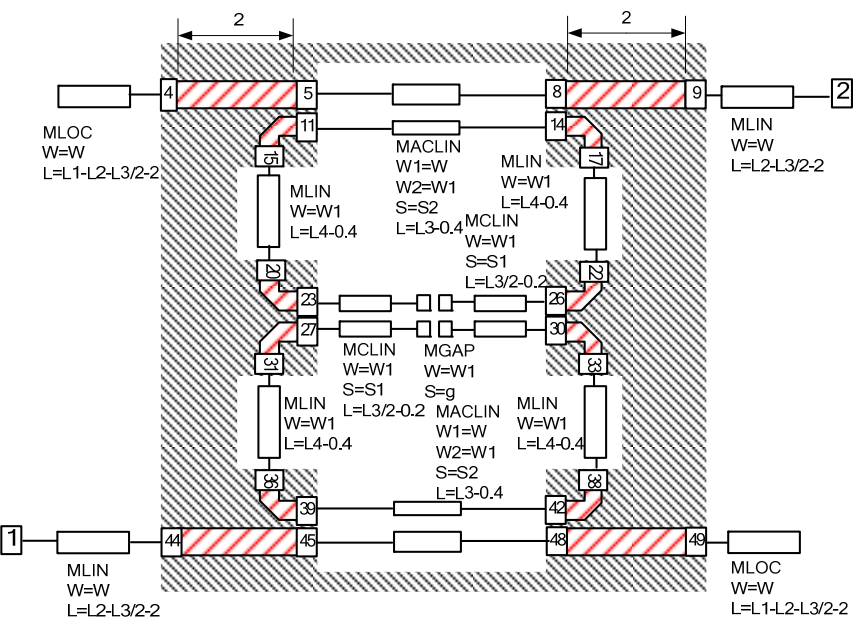

Fig. 9. Open-loop ring resonator bandpass filter in ADS with embedded surrogate elements.

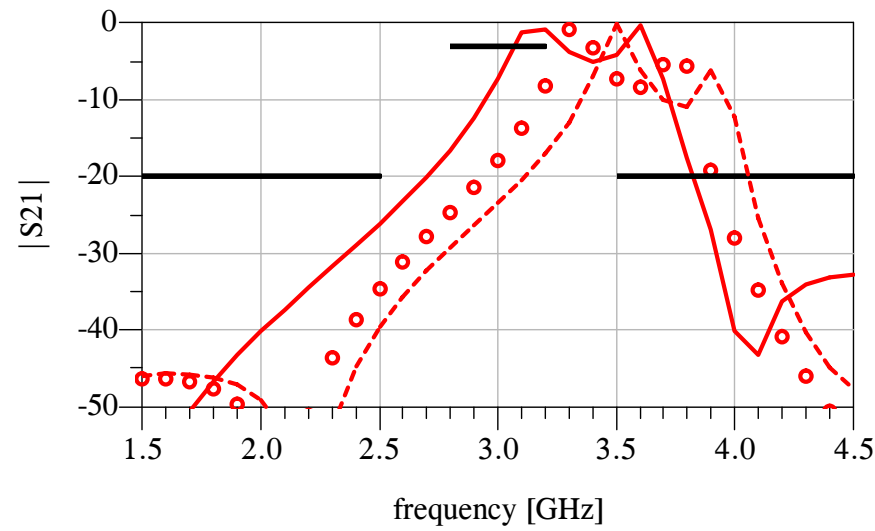

Fig. 10. Initial responses: tuning model $(-)$, fine model $(\circ)$, and fine model with co-calibrated ports (---).

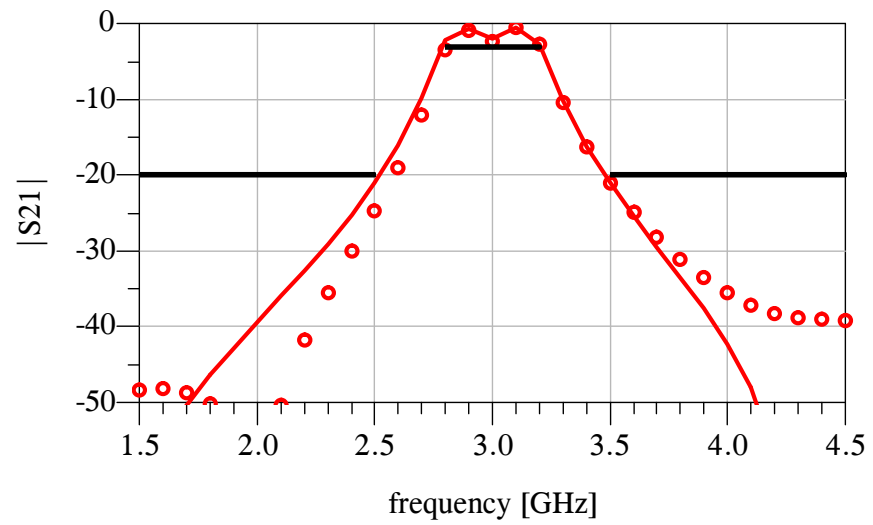

Fig. 11. Responses after two iterations: the tuning model (-) and corresponding fine model $(\circ)$.

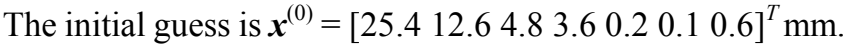
We show the response of our tuning model, the fine model and the fine model with co-calibrated ports in Fig. 10. We compensate the deviation between the tuning model and the fine model by calibrating the preassigned parameters, dielectric constant and substrate height of the surrogate elements.

The tuning model is optimized in ADS with respect to the design parameters. The new design parameters are then assigned to the fine model. The optimal values obtained with (3) are $\boldsymbol{x}^{(0)}=$ [ $\left.\begin{array}{lllllll}20.34 & 7.72 & 6.94 & 2.97 & 0.34 & 0.26 & 0.85\end{array}\right]^{T} \mathrm{~mm}$, after two iterations. The optimized tuning model and the corresponding fine model are shown in Fig. 11.

\section{DISCUSSION}

Our new tuning space mapping methodology falls into the scope of tuning space mapping with analytical calibration, as described in [2], but in its simplest form, the identity function calibration, i.e., parameter conversion is unnecessary.

\section{CONCLUSIONS}

We present a physically-based surrogate element embedding technique to facilitate simulator-based tuning design. It is accurate, since the tuning model is embedded with fine-model couplings and discontinuity information, and aligned with the fine model. The surrogate elements facilitate direct access to the physical design parameters. Our procedure avoids negative tuning parameter values and makes a larger variety of tuning elements possible. It effectively solves the problem of cross-sectional parameter design.

\section{ACKNOWLEDGMENT}

The authors thank Sonnet Software, Inc., Syracuse, NY, for $\boldsymbol{e m}^{\mathrm{TM}}$ and Agilent Technologies, Santa Rosa, CA, for making ADS available.

\section{REFERENCES}

[1] J. Meng, S. Koziel, J.W. Bandler, M.H. Bakr, and Q.S. Cheng, "Tuning space mapping: a novel technique for engineering design optimization," IEEE MTT-S Int. Microwave Symp. Dig., Atlanta, GA, pp 991-994, June 2008.

[2] S. Koziel, J. Meng, J.W. Bandler, M.H. Bakr, and Q.S. Cheng, "Accelerated microwave design optimization with tuning space mapping," IEEE Trans. Microwave Theory Tech., vol. 57, no. 2, pp. 383-394, Feb. 2009.

[3] J.C. Rautio, "RF design closure-companion modeling and tuning methods," IEEE MTT IMS Workshop: Microwave component design using space mapping technology, San Francisco, CA, 2006.

[4] J.C. Rautio, "EM-component-based design of planar circuits," IEEE Microwave Magazine, vol. 8, no. 4, pp. 79-90, Aug. 2007.

[5] J.W. Bandler, Q.S. Cheng, S.A. Dakroury, A.S. Mohamed, M.H. Bakr, K. Madsen, and J. Sondergaard, "Space mapping: the state of the art," IEEE Trans. Microwave Theory Tech., vol. 52, no. 1, pp. 337-361, Jan. 2004.

[6] Q.S. Cheng, J.W. Bandler, and S. Koziel, "Combining coarse and fine models for optimal design," IEEE Microwave Magazine, vol. 9, no. 1, pp. 79-88, Feb. 2008.

[7] D.G. Swanson and R.J. Wenzel, "Fast analysis and optimization of combline filters using FEM," IEEE MTT-S IMS Digest, Boston, MA, pp. 1159-1162, July 2001.

[8] S. Koziel and J.W. Bandler, "Space mapping with multiple coarse models for optimization of microwave components," IEEE Microwave and Wireless Components Letters, vol. 8, no. 1, pp. 1-3, Jan. 2008. 